\title{
CONTACT LENS INNOVATIONS: SPREAD THE WORD
}

\author{
By Chad Rosen, OD, MBA, FAAO \\ Editor-in-Chief
}

Just as we see changes taking place in the health care system, we are also seeing changes take place within the publishing industry. Historically, access to peer-reviewed research and papers has been limited to those who pay membership fees to individual organizations or those working in academic institutions. As health care continues to change and universities are increasing the amount evidence-based medicine taught, a larger emphasis is placed on the scientific method and how to evaluate credible research. For those wanting to expand upon their education, access to scientific research can be difficult to obtain. Openaccess journals have provided a platform in which individuals can easily stay up-to-date on information in his or her specialty.

Many negative connotations have developed around open-access journals. Some publishing companies have been given the term "Predatory Publishers" by the way in which they operate. The Journal of Contact Lens Research \& Science (JCLRS) has been developed to provide a unique forum for clinicians, researchers, and scientists in which original research, case reports, and clinically relevant articles can be shared with optical and ophthalmic professionals all over the world while maintaining the high standards of traditional peer-reviewed journals. One of our main goals is to provide a publication that not only withstands the rigours of vigorous peer review, but it is also to deliver information that is relevant and of value for current and future clinical care. The information located within the publication will be available for anyone to view, creating an opportunity of greater exposure for authors' works than traditional peer-reviewed journals.

The JCLRS believes in transparent operations and has a dedicated editorial board with experience and expertise in various areas of specialty contact lenses. In order to properly represent the international audience of this journal, the board is made up of members from around the globe. Five of the current editors are located in the Americas and three are in Australia. In the process of maintaining a well-rounded International group, additional editors from Asia and Europe are also being consulted. We feel the editorial board, many of which are recognized names in the industry, is the foundation for a respectable journal. Our editors are dedicated to ensuring the integrity of contact lens research. Each accepted article will go through a double-blind peer review process to provide high quality production and maintain thoroughness. The articles will be archived through Portico to preserve any work that has been published and maintain sustainable digital record. In addition, the JCLRS is working on getting indexed with the NLM and Thompson Reuters Web of Science.

Topics for the JCLRS are intended to be centered around specific areas within the contact lens community. They include, but are not limited to: contact lens comfort, multifocal contact lenses, scleral contact lenses, orthokeratology, myopia control, drug-eluting contact lenses, digital and computer chip contact lenses, contact lens materials, and correcting the irregular cornea. Innovations in these areas, among others, can lead to future uses and even create new outlooks for various ocular conditions. Optics continues to be one of those areas with significant progress, with research advancing at an incredible rate. From using customized optics that correct for higher order aberrations in scleral lenses for keratoconus patients, to increased depth of focus lenses for presbyopes, we are seeing technology expanding. Optics also play a major role in myopia control, another increasingly popular topic. Using optics to create contact lenses that provide an adequate mechanism to slow myopia progression has been a primary conversation in many of the national and international conferences. Another key theme revolves around scleral contact lenses and current knowledge of their use. The solution behind

J Cont Lens Res Sci Vol 1(1):1-2; September 20, 2017.

(C) 2017 Journal of Contact Lens Science and Research. All rights reserved. This article is distributed under the terms of the Creative Commons Attribution-Non Commercial 4.0 International License. 
and lens and level of vault has created questions of how much oxygen is actually getting through to the cornea and whether it is detrimental to any abnormal cornea. Long-term goals for patients are to provide healthy options for visual correction with consistent and exceptional comfort.

We have high expectations for the ability of the JCLRS publication to impact readers' decisions when providing clinical care. We hope to display the achievements, challenges and insights of our authors and provide a valid review process through our Editorial Board and Guest Editors. All editors chosen for the $J C L R S$ have demonstrated a passion for contact lenses and have shown a desire to spread new knowledge to as many people as possible so a steady practice of evidence-based care can be obtained. 